International Journal of Applied Mathematics

Volume 31 No. $3 \quad 2018,427-455$

ISSN: $1311-1728$ (printed version); ISSN: $1314-8060$ (on-line version)

doi: http://dx.doi.org/10.12732/ijam.v31i3.10

\title{
LOCAL-IN-TIME SOLVABILITY OF TARGET DETECTION MODEL IN MOLECULAR COMMUNICATION NETWORK
}

\author{
Hirotada Honda \\ 1-7-11 Akabanedai, Kita-Ku, Tokyo \\ 115-0053, JAPAN
}

\begin{abstract}
This paper is concerned with a model of the target detection that is actively discussed in the study of molecular communication network these days. We first verify the solvability of the stationary problem, and then the existence of a strong local-in-time solution to the non-stationary problem in the Sobolev-Slobodetskiı̌ space.
\end{abstract}

AMS Subject Classification: 35K61, 35Q92, 92C17

Key Words: molecular communication; target detection; local-in-time solvability

\section{Introduction}

Molecular communication is one of the most attractive research areas nowadays. It is drastically different from the conventional information communication technologies, and a number of active discussions are held among interdisciplinary researchers, such as biology, mathematics, statistics, information theory, and so forth. It is also closely interacting with another active research area, nano-network.

Molecular communication is expected to possess various applications in engineering, such as bio-engineering, medical, industrial and communications [10].

Among them, the most important application in the medical area is considered to be the drug delivery [10]. There, each bio-nanomachine detects some target area, such as tumor cells in the human body, and release the drug molecules in appropriate area, with suitable volume and timing. Nowadays, many the-

(c) 2018 Academic Publications 
oretical frameworks concerning the method of target detection in molecular communication network are proposed as well as the implementation and experiments in laboratories.

One of the key factors for carrying signals in molecular communication is molecule diffusion in the medium. The early works in this direction are found in [1], [2], and are widely applied in the arguments of coding and encoding methods in the molecular communication these days.

Another key factor of molecular communication is that it enables the cooperation between bio-nanomachines to achieve a certain objective.

For instance, Nakano et al. [11] recently proposed a model of target detection that imposes two different roles on bio-nanomachines: leader and follower. The leader nanomachines search for a target in the human body, and release attractant molecules upon detecting it. Follower nanomachines move according to the concentration gradient of the attractant toward the source of it, and then release drug molecules.

Nakano's model utilizes the chemotaxis of bio-nanomachies. The investigations in such a direction are also found in several papers as [12], [13], and activated the modeling of bio-nanomachines based on the Keller-Segel model [8].

The discussions by Nakano et al. [11] applied the agent-based simulations and experiments, while more theoretical study was conducted by Iwasaki [6] recently.

In it, both the attractant and repellent exist in the model. The attractant makes bio-nanomachies be closer to the target in order to yield the drug molecules there. On the other hand, the repellent contribute to diffusing bionanomachies, and make them search for the target over the broader area. He focused on the concentration of bio-nanomanies, attractant and repellent, and formulated their temporal behavior. The proposed model was a couple of a one-dimensional reaction-diffusion-type partial differential equation and two ordinary differential equations.

It is also to be noted that the proposed model in [6] was based on the variant model of the Keller-Segel equation originally proposed by Okaie [12], but the diffusion terms in equations of attractant and repellent were neglected for simplicity. After showing the existence of a positive stationary solution, Iwasaki numerically verified its stability. However, few theoretical analysis concerning the proposed model were conducted in that paper. In this paper, we rigorously discuss the well-posedness of the model, and show the following issues.

(i) Existence of a non-negative stationary solution is verified;

(ii) The global-in-time solvability of the model proposed by Iwasaki is shown 
under the smallness of data;

(iii) the non-negativeness of the non-stationary solution is shown.

The remaining part of this paper is organized as follows. In the next section, we overview the existing mathematical arguments. In Section 3, we formulate the problem, and in Section 4, we introduce notations used throughout this paper. Main results of this paper are stated in Section 5, followed by their proofs in Section 6.

\section{Existing Arguments}

There exist a number of works concerning the molecular communication network these days, and we limit ourselves to the following arguments those concerning the target detection in the human body. Nakano et al. [11] proposed a mathematical model that describes the temporal behavior of the attractant molecules. They proposed a model in which two roles were imposed on bionanomachines, which they called the leader-follower model. They showed the effectiveness of the proposed method through numerical simulations. They also clarified the situations in which their proposed method worked better than the conventional one, but few mathematically rigorous discussions were held.

Following these works, Iwasaki et al. [6] proposed a mathematical model that concerns the non-diffusion-based mobile molecular communication networks. He focused only on the temporal behavior of the concentration of the attractant, repellent, and bio-nanomachines under the assumption that the concentration of the target is time invariant.

Similar model was discussed in their previous paper [5], including the existence and uniqueness of the solution, and the stability of the stationary solution. It reads

$$
\left\{\begin{array}{l}
\frac{\partial u}{\partial t}=\frac{\partial^{2}}{\partial x^{2}}\left(a u+\alpha u^{2}\right)-\mu \frac{\partial}{\partial x}\left[u \frac{\partial}{\partial x}(T(x) u)\right] \quad \text { in } I \times \mathbf{R}_{+} \\
\frac{\partial u}{\partial x}=0 \quad \text { on } \partial I \times(0, \infty) \\
\left.u\right|_{t=0}=\left(u_{0}, v_{0}, w_{0}\right) \text { on } I .
\end{array}\right.
$$

where and hereafter $I \equiv(0,1), \partial I \equiv\{0\} \bigcup\{1\}$, and $\mathbf{R}_{+} \equiv(0, \infty)$. It was proposed as a simplified version of that proposed by Okaie et al. [12] that 
is similar to the Keller-Siegel model [8] but includes the time variant target concentration:

$$
\left\{\begin{array}{l}
\frac{\partial u}{\partial t}=a_{1} \frac{\partial^{2} u}{\partial x^{2}}-\frac{\partial}{\partial x}\left[u\left(\frac{\partial}{\partial x} \chi_{1}(v)-\frac{\partial}{\partial x} \chi_{2}(w)\right)\right], \\
\frac{\partial v}{\partial t}=a_{2} \frac{\partial^{2} v}{\partial x^{2}}+g_{1} T(x, t) u-d v, \\
\frac{\partial w}{\partial t}=a_{3} \frac{\partial^{2} w}{\partial x^{2}}+g_{2} u-h w \quad \text { in } I \times(0, \infty), \\
\frac{\partial u}{\partial x}=\frac{\partial v}{\partial x}=\frac{\partial w}{\partial x}=0 \quad \text { on } \partial I \times(0, \infty), \\
\left.(u, v, w)\right|_{t=0}=\left(u_{0}, v_{0}, w_{0}\right) \quad \text { on } I .
\end{array}\right.
$$

Iwasaki et al. [5] also argued the stability of the stationary solution of (1) by constructing the Lyapunov function. We also point out that although the model in [5] admits constructing the global-in-time solution without the smallness of the initial data, the method does not apply to the model studied in this paper. As for other arguments concerning the model by Okaie [12], see the review by Iwasaki [5] and the references therein.

The model discussed in this paper is a couple of the reaction-diffusion equation and ordinary differential equations. Recently, Marciniak-Czochra et al. [16] studied the non-stability of such systems under certain conditions. It seems meaningful since the reaction-diffusion equation reflexes the denovo patterns or the Turing instability.

On the other hand, Iwasaki et al. [6] numerically showed the stability of a stationary solution of their model.

Since these models stated so far arise from the Keller-Segel model, we briefly overview mathematical arguments concerning the Keller-Segel equations.

There exist huge number of contributions concerning the mathematical arguments of Keller-Segel equations and its variations (see, for instance, [3] and references therein), and therefore, we limit ourselves to the relational arguments to our problem.

Schaaf [15] studied the stationary solution to the Keller-Segel equation under the general non-linearity, and reduced the problem to a scalar equation by using the bifurcation technique. She also provided a criterion for bifurcation of solution. Osaki and Yagi [14] provided the global-in-time solution of the classical one-dimensional Keller-Segel equation. Later, Hillen and Potapov [3] discussed the one-dimensional chemotaxis model: 


$$
\left\{\begin{array}{l}
u_{t}=u_{x x}-\chi\left(u v_{x}\right)_{x} \\
v_{t}=\varepsilon v_{x x}+u-a v
\end{array}\right.
$$

on a closed interval with homogeneous Neumann or periodic boundary conditions. They constructed the local-in-time solution by virtue of the fixed point theorem (it was argued for the space dimension $n \geq 1$ ), and then constructed the a-priori estimate by using the estimate of the heat kernel. However, this method does not apply to our discussion because in our model depicted in (2) in the next section does not have this term.

The existence and stability of a spike solution to the one-dimensional KellerSegel equation in the asymptotic limit of a large mass was studied by Kang [7]. They also discussed the global-in-time solvability of a reduced version of the Keller-Segel equation. The latter part is conducted by using the energy method.

For reader's convenience, we note that through surveys are provided by Horstmann [4] and references therein.

\section{Formulation}

In this section, we formulate the problem to be discussed in this paper. Due to Iwasaki et al. [6], the temporal behavior of the concentrations of bio-nanomachines, attractant and repellent in one-dimensional space, denoted as $C_{b}(x, t), C_{a}(x, t)$ and $C_{r}(x, t)$, respectively, are represented as follows:

$$
\left\{\begin{array}{l}
\frac{\partial C_{b}}{\partial t}=D_{b} \frac{\partial^{2} C_{b}}{\partial x^{2}}-\frac{\partial}{\partial x}\left[C_{b}\left(V_{a} \frac{\partial C_{a}}{\partial x}-V_{r} \frac{\partial C_{r}}{\partial x}\right)\right] \\
\frac{\partial C_{a}}{\partial t}=a_{1}(x) C_{b}(x, t)-k_{a} C_{a}(x, t), \\
\frac{\partial C_{r}}{\partial t}=a_{2}(x) C_{b}(x, t)-k_{r} C_{r}(x, t) \quad \text { in } I
\end{array}\right.
$$

with boundary and initial conditions

$$
\left\{\begin{array}{l}
\frac{\partial C_{b}}{\partial x}-C_{b}\left(V_{a} \frac{\partial C_{a}}{\partial x}-V_{r} \frac{\partial C_{r}}{\partial x}\right)=0 \quad \text { on } \partial I \\
\boldsymbol{u}(x, 0)=\boldsymbol{u}_{0}(x) \equiv\left(C_{b 0}(x), C_{a 0}(x), C_{r 0}(x)\right)^{\mathrm{T}} \text { on } I .
\end{array}\right.
$$

For the sake of simplicity, we introduced a notation $\boldsymbol{u}=\left(C_{b}, C_{a}, C_{r}\right)^{\mathrm{T}}$ above. Here, $t$ is time, $x$, the location of materials on the tissue surface, $a_{1}(x)=$ 
$g_{a} h(x) /\left(h(x)+K_{a}\right), a_{2}(x)=g_{r} K_{r} /\left(h(x)+K_{r}\right)$ with $h(x)$ being the target concentration, and $K_{a}$ (or $K_{r}$ ), positive constants standing for the target concentration leading to the half maximum attractant (or repellent, resp.) production rate. The notation $g_{a}$ ( or $g_{r}$ ) is also a positive constant representing the maximum attractant (or repellent, resp.) production rate; In [6], the author provided the existence of a stationary solution $\overline{\boldsymbol{u}}(x) \equiv\left(\bar{C}_{b}(x), \bar{C}_{a}(x), \bar{C}_{r}(x)\right)^{\mathrm{T}}$ to (2)-(3) that is subject to:

$$
\left\{\begin{array}{l}
\frac{\mathrm{d}^{2} \bar{C}_{b}}{\mathrm{~d} x^{2}}-\frac{\mathrm{d}}{\mathrm{d} x}\left[\bar{C}_{b}\left(V_{a} \frac{\mathrm{d} \bar{C}_{a}}{\mathrm{~d} x}-V_{r} \frac{\mathrm{d} \bar{C}_{r}}{\mathrm{~d} x}\right)\right]=0, \\
a_{1}(x) \bar{C}_{b}(x)-\bar{C}_{a}(x)=0, \\
a_{2}(x) \bar{C}_{b}(x)-\bar{C}_{r}(x)=0 \quad \text { in } I, \\
\frac{\partial \bar{C}_{b}}{\partial x}-\bar{C}_{b}\left(V_{a} \frac{\partial \bar{C}_{a}}{\partial x}-V_{r} \frac{\partial \bar{C}_{r}}{\partial x}\right)=0 \quad \text { on } \partial I, \\
\int_{I} \bar{C}_{b}(x) \mathrm{d} x=1 .
\end{array}\right.
$$

They also showed the stability of $\overline{\boldsymbol{u}}(x)$, that is, the convergence of the solution of (2)-(3) to that of (4) through numerical simulations under specific values of parameters.

In this paper, we study the well-posedness of (2)-(3). We first subtract $\overline{\boldsymbol{u}}(x)$ from the solution to problem (2)-(3), and consider the problem concerning

$$
\widetilde{\boldsymbol{u}}(x, t) \equiv\left(\widetilde{C}_{b}, \widetilde{C}_{a}, \widetilde{C}_{r}\right)^{\mathrm{T}} \equiv \boldsymbol{u}(x, t)-\overline{\boldsymbol{u}}(x)
$$


It reads

$$
\left\{\begin{array}{l}
\frac{\partial \widetilde{C}_{b}}{\partial t}=D_{b} \frac{\partial^{2} \widetilde{C}_{b}}{\partial x^{2}}-\frac{\partial}{\partial x}\left\{\bar{C}_{b}\left(V_{a} \frac{\partial \widetilde{C}_{a}}{\partial x}-V_{r} \frac{\partial \widetilde{C}_{r}}{\partial x}\right)\right\} \\
\quad-\frac{\partial}{\partial x}\left[\widetilde{C}_{b}\left\{V_{a}\left(\frac{\partial \widetilde{C}_{a}}{\partial x}+\frac{\partial \bar{C}_{a}}{\partial x}\right)-V_{r}\left(\frac{\partial \widetilde{C}_{r}}{\partial x}+\frac{\partial \bar{C}_{r}}{\partial x}\right)\right\}\right] \\
\frac{\partial \widetilde{C}_{a}}{\partial t}=a_{1}(x) \widetilde{C}_{b}(x, t)-k_{a} \widetilde{C}_{a}(x, t), \\
\frac{\partial \widetilde{C}_{r}}{\partial t}=a_{2}(x) \widetilde{C}_{b}(x, t)-k_{r} \widetilde{C}_{r}(x, t) \text { in } I_{T} \\
D_{b} \frac{\partial \widetilde{C}_{b}}{\partial x}-\left[\widetilde{C}_{b}\left\{V_{a}\left(\frac{\partial \widetilde{C}_{a}}{\partial x}+\frac{\partial \bar{C}_{a}}{\partial x}\right)-V_{r}\left(\frac{\partial \widetilde{C}_{r}}{\partial x}+\frac{\partial \bar{C}_{r}}{\partial x}\right)\right\}\right] \\
-\bar{C}_{b}\left(V_{a} \frac{\partial \widetilde{C}_{a}}{\partial x}-V_{r} \frac{\partial \widetilde{C}_{r}}{\partial x}\right)=0 \quad \text { on } \partial I \\
\text { on } I,
\end{array}\right.
$$

where $\widetilde{\boldsymbol{u}}_{0}=\left(\widetilde{C}_{b 0}, \widetilde{C}_{a 0}, \widetilde{C}_{r 0}\right)^{\mathrm{T}}$.

\section{Notations}

In the following, let $T>0, \mathcal{G}$ be an arbitrary open set in $\mathbf{R}, I \equiv(0,1)$ and $I_{T} \equiv I \times(0, T)$. Hereafter, $C^{l}(\mathcal{G})(l \in \mathbf{N})$ stands for sets of all functions defined on $\mathcal{G}$ that have $l$-th order continuous derivatives. We also denote a set of Lipschitz continuous functions with the Lipschitz coefficient $L$ on $\mathcal{G}$ as $C^{(L)}(\mathcal{G})$ with $L \in(0,1) . L_{2}(\mathcal{G})$ means a set of square-integrable functions defined on $\mathcal{G}$, equipped with the norm $\left(L_{2}\right.$-norm)

$$
|f|_{2} \equiv\left(\int_{\mathcal{G}}|f(x)|^{2} \mathrm{~d} x\right)^{\frac{1}{2}} .
$$

The inner product in $L_{2}(\mathcal{G})$ is defined by

$$
\left(f_{1}, f_{2}\right) \equiv \int_{\mathcal{G}} f_{1}(x) \overline{f_{2}(x)} \mathrm{d} x,
$$

where $\bar{z}$ stands for the complex conjugate of $z \in \boldsymbol{C}$.

Likewise, by $|\cdot|_{p}$, we denote the usual $L_{p}$ norm on $I$ : 


$$
|f|_{p} \equiv\left\{\begin{array}{l}
\left(\int_{0}^{1}|f(x)|^{p} \mathrm{~d} x\right)^{\frac{1}{p}}, \quad 1<p<+\infty \\
\operatorname{ess} \sup _{x \in I}|f(x)|, \quad p=+\infty
\end{array}\right.
$$

For a Banach space $B$ with the norm $\|\cdot\|_{B}$, we denote the space of $B$-valued measurable functions $f(t)$ on the interval $(a, b)$ by $L_{p}(a, b ; B)$, whose norm is defined by

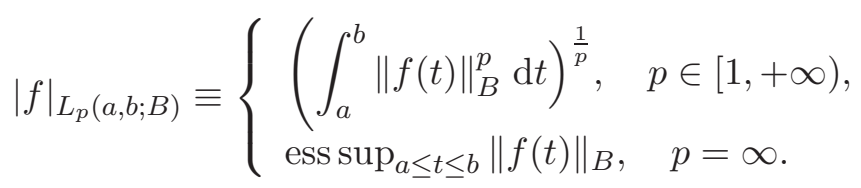

By $W_{2}^{l}(\mathcal{G})(l>0)$, we mean a space of functions $u(x), x \in \mathcal{G}$ equipped with the norm $\|u\|_{W_{2}^{l}(\mathcal{G})}^{2}=\sum_{|\alpha|<l}\left|D^{\alpha} u\right|_{2}^{2}+\|u\|_{\dot{W}_{2}^{l}(\mathcal{G})}^{2}$, where

$$
\left\{\begin{array}{c}
\|u\|_{\dot{W}_{2}^{l}(\mathcal{G})}^{2}=\sum_{|\alpha|=l}\left|D^{\alpha} u\right|_{2}^{2}=\sum_{|\alpha|=l} \int_{\mathcal{G}}\left|D^{\alpha} u(x)\right|^{2} \mathrm{~d} x \quad \text { if } l \text { is an integer, } \\
\|u\|_{\dot{W}_{2}^{l}(\mathcal{G})}^{2}=\sum_{|\alpha|=[l]} \int_{\mathcal{G}} \int_{\mathcal{G}} \frac{\left|D^{\alpha} u(x)-D^{\alpha} u(y)\right|^{2}}{|x-y|^{n+2}\{l\}} \mathrm{d} x \mathrm{~d} y \quad \text { if } l \text { is a non-integer } \\
l=[l]+\{l\}, 0<\{l\}<1 .
\end{array}\right.
$$

Next, for arbitrary $T>0$, we introduce anisotropic Sobolev-Slobodetskiu spaces [18]:

$$
W_{2}^{l, \frac{l}{2}}\left(\mathcal{G}_{T}\right) \equiv W_{2}^{l, 0}\left(\mathcal{G}_{T}\right) \bigcap W_{2}^{0, \frac{l}{2}}\left(\mathcal{G}_{T}\right)\left(\mathcal{G}_{T} \equiv \mathcal{G} \times(0, T)\right)
$$

whose norms are defined by

$$
\begin{aligned}
\|u\|_{W_{2}^{l, \frac{l}{2}}\left(\mathcal{G}_{T}\right)}^{2} & =\int_{0}^{T}\|u(\cdot, t)\|_{W_{2}^{l}(\mathcal{G})}^{2} \mathrm{~d} t+\int_{\mathcal{G}}\|u(x, \cdot)\|_{W_{2}^{\frac{l}{2}}(0, T)}^{2} \mathrm{~d} x \\
& \equiv\|u\|_{W_{2}^{l, 0}\left(\mathcal{G}_{T}\right)}^{2}+\|u\|_{W_{2}^{0, \frac{l}{2}}\left(\mathcal{G}_{T}\right)}^{2}
\end{aligned}
$$

The set of functions with vanishing initial data, $\stackrel{\circ}{W}_{2}^{l, \frac{l}{2}}\left(\mathcal{G}_{T}\right)$ is defined as [9]: 


$$
\stackrel{\circ}{W}_{2}^{l, \frac{l}{2}}\left(\mathcal{G}_{T}\right)=\left\{u \in W_{2}^{l, \frac{l}{2}}\left(\mathcal{G}_{T}\right)\left|\frac{\partial^{k} u}{\partial t^{k}}\right|_{t=0}=0\left(k=0,1,2, \ldots,\left[\frac{l}{2}\right]-1\right)\right\} .
$$

For simplicity, we shall use the following notations:

$$
\begin{aligned}
& W_{u}^{(l)}(T) \equiv W_{2}^{l, \frac{l}{2}}\left(I_{T}\right) \times W_{2}^{l, \frac{l}{2}}\left(I_{T}\right) \times W_{2}^{l, \frac{l}{2}}\left(I_{T}\right), \\
& W_{f}^{(l)}(T) \equiv W_{2}^{l-2, \frac{l-2}{2}}\left(I_{T}\right) \times W_{2}^{l-2, \frac{l-2}{2}}\left(I_{T}\right) \\
& \times W_{2}^{l-2, \frac{l-2}{2}}\left(I_{T}\right) \times W_{2}^{\frac{l}{2}-\frac{3}{4}}(0, T), \\
& \stackrel{\circ}{W}_{u}^{(l)}(T) \equiv \stackrel{\circ}{W}_{2}^{l, \frac{l}{2}}\left(I_{T}\right) \times \stackrel{\circ}{W}_{2}^{l, \frac{l}{2}}\left(I_{T}\right) \times \stackrel{\circ}{W}_{2}^{l, \frac{l}{2}}\left(I_{T}\right), \\
& \stackrel{\circ}{W}_{f}^{(l)}(T) \equiv \stackrel{\circ}{W}_{2}^{l-2, \frac{l-2}{2}}\left(I_{T}\right) \times \stackrel{\circ}{W}_{2}^{l-2, \frac{l-2}{2}}\left(I_{T}\right) \\
& \times \stackrel{\circ}{W}_{2}^{l-2, \frac{l-2}{2}}\left(I_{T}\right) \times \stackrel{\circ}{W}_{2}^{\frac{l}{2}-\frac{3}{4}}(0, T) .
\end{aligned}
$$

We also use notations like

$$
W_{u}^{(l)}\left(t_{1}, t_{2}\right) \equiv W_{2}^{l, \frac{l}{2}}\left(I \times\left(t_{1}, t_{2}\right)\right) \times W_{2}^{l, \frac{l}{2}}\left(I \times\left(t_{1}, t_{2}\right)\right) \times W_{2}^{l, \frac{l}{2}}\left(I \times\left(t_{1}, t_{2}\right)\right) .
$$

The norms of these spaces are denoted in such ways as $\|\cdot\|_{W_{u}^{(l)}(T)}$, and so forth. The norms of product spaces are defined in the usual manner.

Hereafter, $c$ 's with suffixes represent constants in the estimate of some quantities. When we denote $c(t)$ with suffixes, it depends on $t$.

\section{Main Results}

In this section, we state the main results of this paper, and prove it. First, we discuss the solvability of (4).

\subsection{Solvability of Stationary Problem}

In this section, we discuss the solvability of (4). Before discussing the nonstationary problem, we first consider the stationary problem (4). In the followings, $f^{\prime}(x)$ denotes the derivative of a function $f(x)$ with respect to $x$ in general. 
Theorem 1. Assume that $F(x) \equiv V_{a} a_{1}(x)-V_{r} a_{2}(x)$ satisfies $F^{\prime}(x) \neq 0$ on $\bar{I}$, the closure of $I$. We also assume $a_{j} \in C^{2}(\bar{I})(j=1,2), h(x) \in C^{2}(\bar{I})$. Then there exists a unique solution $\left(\bar{C}_{b}, \bar{C}_{a}, \bar{C}_{r}\right) \in C^{2}(I)$ to (4). It also satisfies $\bar{C}_{b} \geq 0$ on $I$.

Iwasaki [5] discussed a similar problem, but their method does not work well here, because they assume the positiveness of the solution from the beginning. Instead, we rely on the standard existence argument of ordinary differential equation.

Proof. Problem $(4)_{1}$ can be written as

$$
D_{b} \bar{C}_{b}^{\prime}-\bar{C}_{b}\left(F \bar{C}_{b}\right)^{\prime}=0
$$

Actually, this holds on $\bar{I}$ by virtue of the boundary conditions $(4)_{4}$. Rewrite (6) as

$$
\left(D_{b}-F \bar{C}_{b}\right) \bar{C}_{b}^{\prime}=F^{\prime} \bar{C}_{b}^{2}
$$

In virtue of the assumption of the theorem, we then have

$$
1-F \bar{C}_{b} \neq 0 \text { on } I \text {. }
$$

Thus, due to the existence theorem of ordinary differential equation yields the desired result. The latter part of the statement is proved as follows. Assume there exists a local minima $x_{0} \in I$ such that $\bar{C}_{b}\left(x_{0}\right)<0$. Then, we have $\bar{C}_{b}^{\prime}\left(x_{0}\right)=0$, and so $F^{\prime}\left(\bar{C}_{b}\left(x_{0}\right)\right)^{2}=0$ due to $(7)$. But this contradicts the assumption. Next, we consider the case $\bar{C}_{b}<0$ and $\bar{C}_{b}^{\prime}<0$ at $x=1$. But then, there exists a certain point $x_{1} \in I$ such that $\bar{C}_{b}\left(x_{1}\right)=0$ and $\bar{C}_{b}\left(x_{1}\right)<0$, which contradicts (7).

The following is the main result of this paper.

Theorem 2. Let $T_{1}>0$ be an arbitrary number, and $l>5 / 2$, and assume

(i) $C_{b 0} \in W_{2}^{l-1}(I), C_{a 0}, C_{r 0} \in W_{2}^{l}(I)$,

(ii) $a_{j}(x) \in C^{2}(\bar{I})(j=1,2)$.

In addition, let the compatibility conditions of order $[(l-1) / 2]$ be satisfied.

Then, there exists a certain $T_{*}>0$ and $\widetilde{\boldsymbol{u}}(x, t)=\left(C_{b}, C_{a}, C_{r}\right)^{\mathrm{T}} \in W_{u}^{(l)}\left(T_{*}\right)$ that satisfies (5) over the time interval $\left(0, T_{*}\right)$. 
In order to prove Theorem 2, we first consider the linear problem. Next, we make use of the multiplicative inequalities and the iteration method to verify the unique existence of a local-in-time solution.

\subsection{Linear Problem}

In this subsection, we first prove the solvability of the linear problem associated with (5), and then Theorem 2. We first consider the following linearized problem.

$$
\left\{\begin{aligned}
& \frac{\partial \widetilde{C}_{b}}{\partial t}-D_{b} \frac{\partial^{2} \widetilde{C}_{b}}{\partial x^{2}}+ \frac{\partial}{\partial x}\left\{\widetilde{C}_{b}\left(V_{a} \frac{\partial \bar{C}_{a}}{\partial x}-V_{r} \frac{\partial \bar{C}_{r}}{\partial x}\right)\right\} \\
&+\frac{\partial}{\partial x}\left\{\bar{C}_{b}\left(V_{a} \frac{\partial \widetilde{C}_{a}}{\partial x}-V_{r} \frac{\partial \widetilde{C}_{r}}{\partial x}\right)\right\}=F_{1} \\
& \frac{\partial \widetilde{C}_{a}}{\partial t}=a_{1}(x) \widetilde{C}_{b}(x, t)-k_{a} \widetilde{C}_{a}(x, t) \\
& \frac{\partial \widetilde{C}_{r}}{\partial t}=a_{2}(x) \widetilde{C}_{b}(x, t)-k_{r} \widetilde{C}_{r}(x, t) \quad \text { in } I \\
& \quad-\bar{C}_{b}\left(V_{a} \frac{\partial \widetilde{C}_{a}}{\partial x}-V_{r} \frac{\partial \widetilde{C}_{r}}{\partial x}\right)=F_{2} \text { on } \partial I \\
& D_{b}-\widetilde{C}_{b}\left(V_{a} \frac{\partial \bar{C}_{a}}{\partial x}-V_{r} \frac{\partial \bar{C}_{r}}{\partial x}\right) \\
& \widetilde{\boldsymbol{u}}(x, 0)=\widetilde{\boldsymbol{u}}_{0}(x) \quad \text { at } t=0
\end{aligned}\right.
$$

where $\widetilde{\boldsymbol{u}}_{0}=\left(\widetilde{C}_{b 0}, \widetilde{C}_{a 0}, \widetilde{C}_{r 0}\right)^{\mathrm{T}}$. We have the following theorem.

Theorem 3. Let $T_{1}>0$ be an arbitrary number, and $l>5 / 2$. We also assume $a_{j} \in C^{2}(\bar{I})(j=1,2), \widetilde{\boldsymbol{u}}_{0} \in W_{2}^{l-1}(I), F_{1} \in W_{2}^{l-2, \frac{l-2}{2}}\left(I_{T_{1}}\right)$, and $F_{2} \in W_{2}^{\frac{l}{2}-\frac{3}{4}}\left(0, T_{1}\right)$. In addition, let the compatibility conditions up to order $[(l-1) / 2]$ be satisfied. Then, there exists a unique solution $\widetilde{\boldsymbol{u}} \in W_{u}^{(l)}\left(T_{1}\right)$ to (8) satisfying

$$
\|\widetilde{\boldsymbol{u}}\|_{W_{u}^{(l)}\left(T_{1}\right)} \leq c_{51}\left(\left\|F_{1}\right\|_{W_{2}^{l-2, \frac{l-2}{2}}\left(I_{T_{1}}\right)}+\left\|F_{2}\right\|_{W_{2}^{\frac{l}{2}-\frac{3}{4}}\left(0, T_{1}\right)}+\left\|\widetilde{\boldsymbol{u}}_{0}\right\|_{W_{2}^{l-1}(I)}\right) .
$$

Since the sufficient compatibility conditions are satisfied, we first consider (8) with vanishing initial data. More precisely, introducing notations $\boldsymbol{f}_{1}=$ 
$\left(F_{1}, 0,0\right)^{\mathrm{T}}$

$$
L\left(x, \frac{\partial}{\partial x}\right) \widetilde{\boldsymbol{u}}=\left[\begin{array}{c}
D_{b} \frac{\partial^{2} \widetilde{C}_{b}}{\partial x^{2}}-\frac{\partial}{\partial x}\left\{\widetilde{C}_{b}\left(V_{a} \frac{\partial \bar{C}_{a}}{\partial x}-V_{r} \frac{\partial \bar{C}_{r}}{\partial x}\right)\right\} \\
\quad-\frac{\partial}{\partial x}\left\{\bar{C}_{b}\left(V_{a} \frac{\partial \widetilde{C}_{a}}{\partial x}-V_{r} \frac{\partial \widetilde{C}_{r}}{\partial x}\right)\right\} \\
a_{1}(x) \widetilde{C}_{b}(x, t)-k_{a} \widetilde{C}_{a}(x, t) \\
a_{2}(x) \widetilde{C}_{b}(x, t)-k_{r} \widetilde{C}_{r}(x, t)
\end{array}\right],
$$

we construct a sequence of functions $\boldsymbol{u}^{(j)}(j=0,1,2, \ldots,[l / 2])$ in the following manner:

$$
\begin{aligned}
\boldsymbol{u}^{(0)}(x) & =\widetilde{\boldsymbol{u}}_{0}(x), \boldsymbol{u}^{(1)}(x)=L\left(x, \frac{\partial}{\partial x}\right) \widetilde{\boldsymbol{u}}_{0}(x)+\boldsymbol{f}_{1}(x, 0), \\
\boldsymbol{u}^{(j+1)}(x)= & \frac{\partial^{j}}{\partial t^{j}} L\left(x, \frac{\partial}{\partial x}\right) \boldsymbol{u}+\left.\frac{\partial^{j} \boldsymbol{f}_{1}}{\partial t^{j}}\right|_{t=0} \\
& =L\left(x, \frac{\partial}{\partial x}\right) \boldsymbol{u}^{(j)}+\left.\frac{\partial^{j} \boldsymbol{f}_{1}}{\partial t^{j}}\right|_{t=0}(j=2,3, \ldots,[l / 2]) .
\end{aligned}
$$

As we will see in Section 5.3, this $\left\{\boldsymbol{u}^{(j)}\right\}_{j}$ satisfies

$$
\begin{gathered}
\left\|\boldsymbol{u}^{(j)}\right\|_{W_{2}^{l-1-2 j}(I)} \leq c_{510}\left(\left\|\boldsymbol{f}_{1}\right\|_{W_{2}^{l-2, \frac{l-2}{2}}\left(I_{T_{1}}\right)}+\left\|\widetilde{\boldsymbol{u}}_{0}\right\|_{W_{2}^{l-1}(I)}\right) \\
(j=0,1,2, \ldots,[l / 2]) .
\end{gathered}
$$

We then construct a function $\boldsymbol{v}(x, t) \in W_{2}^{l, \frac{l}{2}}\left(\mathbf{R}_{T_{1}}\right)$ which satisfies

$$
\left.\frac{\partial^{j} \boldsymbol{v}}{\partial t^{j}}\right|_{t=0}=\boldsymbol{u}^{(j)} \quad(j=0,1,2, \ldots,[l / 2]),
$$


and define $\breve{\boldsymbol{u}}=\widetilde{\boldsymbol{u}}-\boldsymbol{v}$. Obviously $\breve{\boldsymbol{u}}$ satisfies

$$
\left\{\begin{array}{l}
\frac{\partial \breve{\boldsymbol{u}}}{\partial t}-L\left(x, \frac{\partial}{\partial x}\right) \breve{\boldsymbol{u}}=\boldsymbol{f}_{1}^{\prime} \quad \text { in } I \\
B\left(x, \frac{\partial}{\partial x}\right) \breve{\boldsymbol{u}}=F_{2}^{\prime} \quad \text { on } \partial I \\
\left.\breve{\boldsymbol{u}}\right|_{t=0}=\mathbf{0} \quad \text { on } I
\end{array}\right.
$$

where

$$
\boldsymbol{f}_{1}^{\prime} \equiv\left(F_{1}^{\prime}, 0,0\right)^{\mathrm{T}}=\boldsymbol{f}_{1}-L\left(x, \frac{\partial}{\partial x}\right) \boldsymbol{v}, \quad F_{2}^{\prime}=F_{2}-B\left(x, \frac{\partial}{\partial x}\right) \boldsymbol{v} .
$$

To $(8)^{\prime}$, we advocate

Theorem 4. Let $T_{1}>0$ be an arbitrary number, $l>5 / 2$, and let us assume $a_{j} \in C^{2}(\bar{I})(j=1,2), F_{1} \in \stackrel{\circ}{W}_{2}^{l-2, \frac{l-2}{2}}\left(I_{T_{1}}\right)$, and $F_{2} \in \stackrel{\circ}{W}_{2}^{\frac{l}{2}-\frac{3}{4}}\left(0, T_{1}\right)$. In addition, let the compatibility conditions of order $[(l-1) / 2]$ be satisfied. Then, there exists a number $\tau_{0}>0$, dependent on $a_{j}(j=1,2), \bar{C}_{b}, \bar{C}_{a}$ and $\bar{C}_{r}$, and a unique solution $\breve{\boldsymbol{u}} \in \stackrel{\circ}{W}_{u}^{(l)}\left(\tau_{0}\right)$ to $(8)^{\prime}$ satisfying

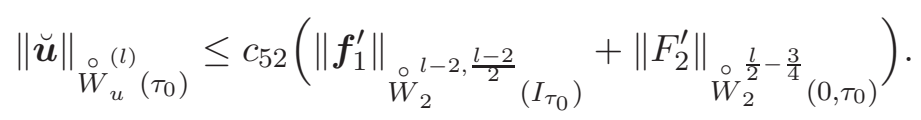

Since $(8)^{\prime}$ consists of equations and boundary conditions with variable coefficients, we appeal to the method of regularizer [9].

Let $\mathcal{A}$ be a linear operator defined on $\stackrel{\circ}{W}_{u}^{(l)}\left(T_{1}\right)$, that associates $\breve{\boldsymbol{u}}$ with $\left(\frac{\partial \breve{\boldsymbol{u}}}{\partial t}-L \breve{\boldsymbol{u}}, B \breve{\boldsymbol{u}}\right)$. It is obvious that $\mathcal{A}$ is bounded. Then, (8)' is interpreted as an abstract problem

$$
\mathcal{A} \breve{u}=f
$$

for $\boldsymbol{f}=\left(F_{1}, 0,0, F_{2}\right)^{\mathrm{T}} \in \stackrel{\circ}{W}_{f}^{(l)}\left(T_{1}\right)$. Therefore, we have to show the existence of the bounded inverse operator $\mathcal{A}^{-1}$. In the sequel, we construct an operator $\mathcal{R}$ that acts from $\stackrel{\circ}{W}_{f}^{(l)}\left(T_{1}\right)$ to $\stackrel{\circ}{W}_{u}^{(l)}\left(T_{1}\right)$, and that satisfies

$$
\begin{aligned}
& \mathcal{A R} \boldsymbol{f}=\boldsymbol{f}+\mathcal{S} \boldsymbol{f}, \\
& \mathcal{R} \mathcal{A} \breve{\boldsymbol{u}}=\breve{\boldsymbol{u}}+\mathcal{E} \breve{\boldsymbol{u}},
\end{aligned}
$$


for any $\boldsymbol{f} \in \stackrel{\circ}{W}_{f}^{(l)}\left(T_{1}\right)$ and $\boldsymbol{u} \in \stackrel{\circ}{W}_{u}^{(l)}\left(T_{1}\right)$, where $\mathcal{S}$ and $\mathcal{E}$ are bounded operators in spaces $\stackrel{\circ}{W}_{f}^{(l)}\left(T_{1}\right)$ and $\stackrel{\circ}{W}_{u}^{(l)}\left(T_{1}\right)$, respectively. We will show that their norms become small if we take $T_{1}$ sufficiently small. The overview of the proof reads as follows.

(i) We construct a covering of $I$;

(ii) In each piece of the covering, we consider the model problem in the whole or half-lines;

(iii) We construct operators $\mathcal{S}$ and $\mathcal{E}$ above, and show the smallness of their norms under small $T_{1}$;

(iv) We show the existence of $\mathcal{A}^{-1}$ under small $T_{1}$.

\subsubsection{Covering of $I$}

We first introduce a covering of $I$, on which we construct the regularizer [9]. Let us introduce two systems of coverings of $I$, say, $\left\{\omega^{(k)}\right\}$ and $\left\{\Omega^{(k)}\right\}$. They are constructed for any small number $\lambda \in(0,1)$ satisfying:

(i) $\omega^{(k)} \subset \Omega^{(k)} \subset \Omega \quad \forall k, \bigcup_{k} \omega^{(k)}=\bigcup_{k} \Omega^{(k)}=I ;$

(ii) For any $\xi \in \Omega$, there exists $\omega^{(k)}$ such that $y \in \omega^{(k)}$ and $\operatorname{dist}\left(x, I \backslash \omega^{(k)}\right) \geq$ $\beta_{1} \lambda$ with a certain $\beta_{1}>0$. Here, the notation $\operatorname{dist}(x, A)$ is the distance between a point $x \in \Omega$ and a set $A \subset \Omega$ in general;

(iii) There exists a natural number $N_{0}$ independent of $\lambda$ such that $\bigcap_{k=1}^{N_{0}+1} \Omega^{(k)}=$ $\phi$

(iv) If $\Omega^{(k)} \cap \partial I=\phi$, then $\omega^{(k)}$ and $\Omega^{(k)}$ are the open intervals with the same center $\xi^{(k)}$ and with the length of their intervals equal to $\frac{\lambda}{2}$ and $\lambda$, respectively. In this case, we represent $k \in \mathcal{N}_{1}$;

(v) In case $\Omega^{(k)} \bigcap\{x=0\} \neq \phi$, we represent $k \in \mathcal{N}_{2}$;

(vi) In case $\Omega^{(k)} \bigcap\{x=1\} \neq \phi$, we represent $k \in \mathcal{N}_{3}$. 
Next, let us introduce a smooth partition of unity $\left\{\zeta^{(k)}(x)\right\}$ subordinated to $\left\{\omega^{(k)}\right\}$ and $\left\{\Omega^{(k)}\right\}$ having the following properties for $\alpha \geq 0$ :

$$
0 \leq \zeta^{(k)}(x) \leq 1, \quad\left|\frac{\mathrm{d}^{\alpha}}{\mathrm{d} x^{\alpha}} \zeta^{(k)}(x)\right| \leq \frac{c_{\alpha}}{\lambda^{\alpha}}, \quad \zeta^{(k)}(x)= \begin{cases}1 & \text { for } x \in \omega^{(k)}, \\ 0 & \text { for } x \in I \backslash \Omega^{(k)},\end{cases}
$$

with some constant $c_{\alpha}$ independent of $k$ and $\lambda$. By virtue of the property (iii) of $\Omega^{(k)}, 1 \leq \sum_{k}\left(\zeta^{(k)}(x)\right)^{2} \leq N_{0}$, and it is possible to take the functions

$$
\eta^{(k)}(x) \equiv \frac{\zeta^{(k)}(x)}{\left(\sum_{j} \zeta^{(j)}(x)\right)^{2}}, \quad k \in \bigcup_{i=1}^{3} \mathcal{N}_{i}
$$

having the properties

$$
\begin{gathered}
\eta^{(k)}(x)=0 \quad \text { in } \quad I \backslash \Omega^{(k)}, \\
\left|\frac{\mathrm{d}^{\alpha}}{\mathrm{d} x^{\alpha}} \eta^{(k)}(x)\right| \leq \frac{c_{\alpha}}{\lambda^{\alpha}} \quad(\alpha=1,2, \ldots), \\
\sum_{k} \eta^{(k)}(x) \zeta^{(k)}(x)=1 .
\end{gathered}
$$

\subsubsection{Problems in whole and Half-Lines}

Now we consider the model problems in the whole and half-lines for each affiliation of $k$. In the followings, let $T_{1}$ be an arbitrary positive number. We introduce following notations to represent the coordinate system of coefficients and the derivatives of operators explicitly:

$$
\begin{gathered}
L_{0}\left(x, \frac{\partial}{\partial x}\right) \widetilde{\boldsymbol{u}} \equiv\left(D_{b} \frac{\partial^{2} \widetilde{C}_{b}}{\partial x^{2}}, a_{1}(x) \widetilde{C}_{b}-k_{a} \widetilde{C}_{a}, a_{2}(x) \widetilde{C}_{b}-k_{r} \widetilde{C}_{r}\right) \\
B_{0}\left(x, \frac{\partial}{\partial x}\right) \widetilde{\boldsymbol{u}}=D_{b} \frac{\partial \widetilde{C}_{b}}{\partial x}-\widetilde{C}_{b}\left(V_{a} \frac{\partial \bar{C}_{a}}{\partial x}-V_{r} \frac{\partial \bar{C}_{r}}{\partial x}\right) .
\end{gathered}
$$

First, we show the case $k \in \mathcal{N}_{1}$. We consider the problem

$$
\left\{\begin{array}{l}
\frac{\partial \widetilde{\boldsymbol{u}}^{(k)}}{\partial t}-L_{0}\left(\xi^{(k)}, \frac{\partial}{\partial x}\right) \widetilde{\boldsymbol{u}}^{(k)}=\boldsymbol{f}_{1}^{(k)} \text { in } \mathbf{R} \\
\left.\widetilde{\boldsymbol{u}}^{(k)}\right|_{t=0}=\mathbf{0} \quad \text { on } \mathbf{R}
\end{array}\right.
$$


for a certain $\xi^{(k)} \in I$ and $\boldsymbol{f}_{1}^{(k)}=\zeta^{(k)} \boldsymbol{f}_{1} \in \stackrel{\circ}{W}_{f}^{(l)}\left(T_{1}\right)$ with $\boldsymbol{f}_{1}=\left(F_{1}, 0,0\right)^{\mathrm{T}}$. It is an elementary fact that the problem (13) has a unique solution $\widetilde{\boldsymbol{u}}^{(k)} \in \stackrel{\circ}{W}_{u}^{(l)}\left(T_{1}\right)$ [9]. Hereafter, we denote by $R^{(k)}$ the operator that corresponds this solution $\widetilde{\boldsymbol{u}}^{(k)}$ to $\boldsymbol{f}_{1}^{(k)}$ in (13).

Next, we consider the case $k \in \mathcal{N}_{2}$. We consider the following initial boundary value problem in the half-line:

$$
\left\{\begin{array}{l}
\frac{\partial \widetilde{\boldsymbol{u}}^{(k)}}{\partial t}-L_{0}\left(0, \frac{\partial}{\partial x}\right) \widetilde{\boldsymbol{u}}^{(k)}=\boldsymbol{f}_{1}^{(k)} \quad \text { in } \mathbf{R}_{(+)}, \\
B_{0}\left(0, \frac{\partial}{\partial x}\right) \widetilde{\boldsymbol{u}}^{(k)}=F_{2}^{(k)} \equiv \zeta^{(k)} F_{2} \quad \text { on } x=0, \\
\left.\widetilde{\boldsymbol{u}}^{(k)}\right|_{t=0}=\mathbf{0} \quad \text { on } I .
\end{array}\right.
$$

This problem also has a unique solution $\widetilde{\boldsymbol{u}}^{(k)} \in \stackrel{\circ}{W}_{u}^{(l)}\left(T_{1}\right)$ by applying the Fourier-Laplace transform [9], and we denote by $R^{(k)}$ the operator that associates with $\boldsymbol{f}^{(k)}=\left(\boldsymbol{f}_{1}^{(k)}, F_{2}^{(k)}\right) \in \stackrel{\circ}{W_{f}^{(l)}}\left(T_{1}\right)$ the solution of the problem (15).

In a similar manner, we define $R^{(k)}$ for $k \in \mathcal{N}_{3}$ that associates with $\boldsymbol{f}^{(k)}=$ $\left(\boldsymbol{f}_{1}^{(k)}, F_{2}^{(k)}\right) \in \stackrel{\circ}{W}_{f}^{(l)}\left(T_{1}\right)$ the solution of the problem (15) with the region relaced by $(-\infty, 1)$.

Now define an operator $\mathcal{R}$, which associates $\boldsymbol{f}=\left(\boldsymbol{f}_{1}, F_{2}\right) \in \stackrel{\circ}{\mathrm{o}_{f}^{(l)}}\left(T_{1}\right)$ with the following items:

$$
\mathcal{R H}=\sum_{k} \eta^{(k)}(x) \widetilde{\boldsymbol{u}}^{(k)}(x, t),
$$

Hereafter we use notations

$$
\widetilde{\boldsymbol{u}}^{(k)}(x, t)=\left\{\begin{array}{l}
R^{(k)} \zeta^{(k)} \boldsymbol{f}_{1} \quad\left(k \in \mathcal{N}_{1}\right), \\
R^{(k)}\left(\zeta^{(k)} \boldsymbol{f}_{1}, \zeta^{(k)} F_{2}\right) \quad\left(k \in \mathcal{N}_{2} \cup \mathcal{N}_{3}\right) .
\end{array}\right.
$$

By virtue of the classical result of the half-space problem, we have the estimates

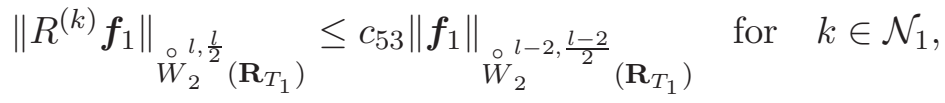

$$
\begin{aligned}
& \left\|R^{(k)}\left(\boldsymbol{f}_{1}, f_{2}\right)\right\|_{\substack{o \\
W_{2}}, \frac{l}{2}}\left(\mathbf{R}_{+} \times\left(0, T_{1}\right)\right)
\end{aligned}
$$

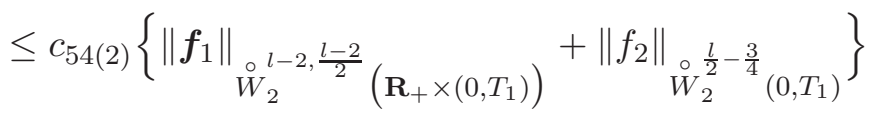




$$
\begin{aligned}
& \text { for } k \in \mathcal{N}_{2} \text {, } \\
& \left\|R^{(k)}\left(\boldsymbol{f}_{1}, f_{2}\right)\right\|_{W_{2}^{\circ}, \frac{l}{2}}\left((-\infty, 1) \times\left(0, T_{1}\right)\right) \\
& \leq c_{54(3)}\left\{\left\|\boldsymbol{f}_{1}\right\|_{\stackrel{\circ}{l}_{2}^{l-2, \frac{l-2}{2}}} \underset{\left((-\infty, 1) \times\left(0, T_{1}\right)\right)}{ }+\left\|f_{2}\right\|_{\stackrel{\circ}{2}_{2}^{\frac{l}{2}-\frac{3}{4}}} \underset{\left(0, T_{1}\right)}{ }\right\} \\
& \text { for } k \in \mathcal{N}_{3} \text {. }
\end{aligned}
$$

By making use of (16)-(18), we easily obtain the following

Lemma 1. For arbitrary $T_{1}>0, \mathcal{R}$ is a bounded operator acting from the space $\stackrel{\circ}{W}_{f}^{(l)}\left(T_{1}\right)$ into the space $\stackrel{\circ}{W}_{u}^{(l)}\left(T_{1}\right)$, and is subjected to the estimate

$$
\|\mathcal{R} \boldsymbol{f}\|_{W_{u}^{\circ}(l)}^{\left(T_{1}\right)} \leq c_{55}\|\boldsymbol{f}\|_{W_{f}^{(l)}\left(T_{1}\right)} .
$$

Now we construct operators $\mathcal{S}$ and $\mathcal{E}$ which are shown to be bounded on the spaces $\stackrel{\circ}{W}_{f}^{(l)}\left(T_{1}\right)$ and $\stackrel{\circ}{W}_{u}^{(l)}\left(T_{1}\right)$, respectively. We define

$$
\mathcal{A}_{0} \widetilde{\boldsymbol{u}}=\left(L_{0} \widetilde{\boldsymbol{u}},\left.B_{0} \widetilde{\boldsymbol{u}}\right|_{\partial I}\right)^{\mathrm{T}}, \quad \mathcal{A}_{1} \widetilde{\boldsymbol{u}}=\left(L_{1} \widetilde{\boldsymbol{u}},\left.B_{1} \widetilde{\boldsymbol{u}}\right|_{\partial I}\right)^{\mathrm{T}},
$$

where $B_{1}=B-B_{0}$. It is to be noted that

$$
\begin{aligned}
\left(\frac{\partial}{\partial t}-L_{0}\left(x, \frac{\partial}{\partial x}\right)\right) \mathcal{R} \boldsymbol{f} & =\sum_{k}\left[\left(\frac{\partial}{\partial t}-L_{0}\left(x, \frac{\partial}{\partial x}\right)\right) \eta^{(k)} \widetilde{\boldsymbol{u}}^{(k)}\right] \\
=\sum_{k} \eta^{(k)} \frac{\partial \widetilde{\boldsymbol{u}}^{(k)}}{\partial t}- & \sum_{k}\left[L_{0}\left(x, \frac{\partial}{\partial x}\right) \eta^{(k)} \widetilde{\boldsymbol{u}}^{(k)}-\eta^{(k)} \mathcal{L}_{0}\left(x, \frac{\partial}{\partial x}\right) \widetilde{\boldsymbol{u}}^{(k)}\right] \\
& -\sum_{k} \eta^{(k)}\left[L_{0}\left(x, \frac{\partial}{\partial x}\right)-L_{0}\left(\xi^{(k)}, \frac{\partial}{\partial x}\right)\right] \widetilde{\boldsymbol{u}}^{(k)} \\
& -\sum_{k} \eta^{(k)} L_{0}\left(\xi^{(k)}, \frac{\partial}{\partial x}\right) \widetilde{\boldsymbol{u}}^{(k)} \\
=F_{1}+\mathcal{S}_{1} \boldsymbol{f}, &
\end{aligned}
$$

where

$$
\begin{aligned}
\mathcal{S}_{1} \boldsymbol{f} \equiv-\sum_{k}\left[L_{0}\left(x, \frac{\partial}{\partial x}\right) \eta^{(k)} \widetilde{\boldsymbol{u}}^{(k)}-\eta^{(k)} L_{0}\left(x, \frac{\partial}{\partial x}\right) \widetilde{\boldsymbol{u}}^{(k)}\right] \\
-\sum_{k} \eta^{(k)}\left[L_{0}\left(x, \frac{\partial}{\partial x}\right)-L_{0}\left(\xi^{(k)}, \frac{\partial}{\partial x}\right)\right] \widetilde{\boldsymbol{u}}^{(k)} .
\end{aligned}
$$


In analogous way, we have

$$
B \mathcal{R} \boldsymbol{f}=F_{2}+\mathcal{S}_{2} \boldsymbol{f}
$$

where

$$
\begin{aligned}
\left.\mathcal{S}_{2} \boldsymbol{f} \equiv B_{1} \mathcal{R} \boldsymbol{f}\right|_{\partial I} & +\left.\sum_{k \in \mathcal{N}_{2}}\left(B_{0} \eta^{(k)} \widetilde{\boldsymbol{u}}^{(k)}-\eta^{(k)} B_{0} \widetilde{\boldsymbol{u}}^{(k)}\right)\right|_{\partial I} \\
& +\left.\sum_{k \in \mathcal{N}_{2}} \eta^{(k)}\left[B_{0}\left(x, \frac{\partial}{\partial x}\right)-B_{0}\left(\xi^{(k)}, \frac{\partial}{\partial x}\right)\right] \widetilde{\boldsymbol{u}}^{(k)}\right|_{\partial I}
\end{aligned}
$$

Thus, (11) holds with

$$
\mathcal{S} \boldsymbol{f}=\left(\mathcal{S}_{1} \boldsymbol{f}, \mathcal{S}_{2} \boldsymbol{f}\right)^{\mathrm{T}} .
$$

Take $T_{1}=\lambda \chi^{2}$ with a certain number $\chi>0$. Then, by combining (16)-(20), we have the estimate [9]

$$
\|S \boldsymbol{f}\|_{\stackrel{\circ}{f}_{f}\left(T_{1}\right)} \leq c_{56} \phi_{01}(\chi, \lambda)\|\boldsymbol{f}\|_{W_{f}\left(T_{1}\right)},
$$

where $\phi_{01}$ is a homogeneous polynomial of its argument.

In a similar manner, we have (13) with

$$
\begin{aligned}
\mathcal{E} \breve{\boldsymbol{u}} \equiv & \mathcal{R} \mathcal{A}_{1} \widetilde{\boldsymbol{u}}+\sum_{k \in \mathcal{N}_{1}} \eta^{(k)} R^{(k)}\left[L_{0}\left(\xi^{(k)}, \frac{\partial}{\partial x}\right) \zeta^{(k)} \breve{\boldsymbol{u}}-\zeta^{(k)} L_{0}\left(\xi^{(k)}, \frac{\partial}{\partial x}\right) \breve{\boldsymbol{u}}\right] \\
& +\sum_{k \in \mathcal{N}_{1}} \eta^{(k)} R^{(k)} \zeta^{(k)}\left[\left\{L_{0}\left(\xi^{(k)}, \frac{\partial}{\partial x}\right)-L_{0}\left(x, \frac{\partial}{\partial x}\right)\right\} \widetilde{\boldsymbol{u}}\right] \\
& +\sum_{k \in \mathcal{N}_{2} \cup \mathcal{N}_{3}} \eta^{(k)} R^{(k)}\left[L_{0}\left(\xi^{(k)}, \frac{\partial}{\partial x}\right) \zeta^{(k)} \breve{\boldsymbol{u}}-\zeta^{(k)} L_{0}\left(\xi^{(k)}, \frac{\partial}{\partial x}\right) \breve{\boldsymbol{u}}\right. \\
& +\sum_{k \in \mathcal{N}_{2} \cup \mathcal{N}_{3}} \eta^{(k)} R^{(k)} \zeta^{(k)}\left[\left\{L_{0}\left(\xi^{(k)}, \frac{\partial}{\partial x}\right)-L_{0}\left(x, \frac{\partial}{\partial x}\right)\right\} \breve{\boldsymbol{u}}, B_{0} \zeta_{0}^{(k)} \breve{\boldsymbol{u}}\right] .
\end{aligned}
$$

Then, by virtue of Lemma 1, we easily have the estimate

$$
\|\mathcal{E} \widetilde{\boldsymbol{u}}\|_{W_{u}^{(l)}\left(T_{1}\right)} \leq c_{57} \phi_{02}(\lambda, \chi)\|\widetilde{\boldsymbol{u}}\|_{W_{W_{u}(l)}^{(l)}\left(T_{1}\right)}
$$


with a homogeneous polynomial $\phi_{02}$. Let us show a part of the estimate. For instance, we have

$$
\begin{gathered}
\left\|L_{0}\left(\xi^{(k)}, \frac{\partial}{\partial x}\right) \zeta^{(k)} \breve{\boldsymbol{u}}-\zeta^{(k)} L_{0}\left(\xi^{(k)}, \frac{\partial}{\partial x}\right) \breve{\boldsymbol{u}},\right\|_{W_{2}^{l-2 \cdot \frac{l-2}{2}}}\left(I_{T_{1}}\right) \\
\leq c_{58}\left(\lambda^{-2}+\lambda^{-1}\right)\left(\varepsilon+C_{\varepsilon} T_{1}\right)\left\|\breve{C}_{b}\right\|_{W_{2}^{l, \frac{l}{2}}\left(I_{T_{1}}\right)} \cdot
\end{gathered}
$$

This is derived from the fact

$$
\begin{aligned}
L_{0}\left(\xi^{(k)}, \frac{\partial}{\partial x}\right) \zeta^{(k)} \breve{\boldsymbol{u}} & -\zeta^{(k)} L_{0}\left(\xi^{(k)}, \frac{\partial}{\partial x}\right) \breve{\boldsymbol{u}} \\
& =\left[\frac{\mathrm{d}^{2} \zeta^{(k)}}{\mathrm{d} x^{2}} \breve{C}_{b}+\frac{\mathrm{d} \zeta^{(k)}}{\mathrm{d} x} \frac{\mathrm{d} \breve{C}_{b}}{\mathrm{~d} x}, 0,0\right]^{\mathrm{T}}
\end{aligned}
$$

and the estimate

$$
\left\|\frac{\mathrm{d}^{2} \zeta^{(k)}}{\mathrm{d} x^{2}} \breve{C}_{b}\right\|_{W_{2}^{l-2, \frac{l-2}{2}}\left(I_{T_{1}}\right)} \leq c_{59}\left\|\frac{\mathrm{d}^{2} \zeta^{(k)}}{\mathrm{d} x^{2}}\right\|_{W_{2}^{l, \frac{l}{2}}\left(I_{T_{1}}\right)}\left\|\breve{C}_{b}\right\|_{W_{2}^{l-2, \frac{l-2}{2}}\left(I_{T_{1}}\right)},
$$

for instance. The estimates (21)-(22) lead us to conclude that both $\mathcal{S}$ and $\mathcal{E}$ are bounded operators in the spaces $\stackrel{\circ}{W}_{f}^{(l)}\left(T_{1}\right)$ and $\stackrel{\circ}{W}_{u}^{(l)}\left(T_{1}\right)$, respectively, and their norms are small if we take $T_{1}$ sufficiently small. Now, it is possible to make the operator norms of $\mathcal{S}$ and $\mathcal{E}$ satisfy

$$
\|\mathcal{S}\|_{\mathcal{L}\left(\stackrel{\circ}{W}_{f}^{(l)}\left(T_{1}\right) ; \stackrel{\circ}{W}_{f}^{(l)}\left(T_{1}\right)\right)}<1, \quad\|\mathcal{E}\|_{\mathcal{L}\left(\stackrel{\circ}{W}_{u}^{(l)}\left(T_{1}\right) ; \stackrel{\circ}{W}_{u}^{(l)}\left(T_{1}\right)\right)}<1
$$

by taking $T_{1}$ small enough, where

$$
\|\cdot\|_{\mathcal{L}\left(W_{1} ; W_{2}\right)}
$$

is the operator norm of a linear operator from a function space $W_{1}$ into $W_{2}$ in general.

Then, by virtue of the contraction mapping principle, it is possible to conclude that there exist $(I+\mathcal{S})^{-1}$ and $(I+\mathcal{E})^{-1}$. By replacing $f$ in $(12)$ by $(I+\mathcal{S})^{-1} \boldsymbol{f}$, and applying $(I+\mathcal{E})^{-1}$ to both sides of $(13)$, we have

$$
\begin{aligned}
\mathcal{A R}(I+\mathcal{S})^{-1} \boldsymbol{f} & =\boldsymbol{f}, \\
(I+\mathcal{E})^{-1} \mathcal{R} A \widetilde{\boldsymbol{u}} & =\widetilde{\boldsymbol{u}} .
\end{aligned}
$$

This means that $\mathcal{A}^{-1}=\mathcal{R}(I+\mathcal{S})^{-1}=(I+\mathcal{E})^{-1} \mathcal{R}$ exists on the time interval $\left(0, T_{1}\right)$. This is equivalent to the boundedness of the operator $\mathcal{A}^{-1}$, which is obvious by virtue of the estimate 


$$
\begin{aligned}
& \left\|\mathcal{A}^{-1}\right\|_{\mathcal{L}\left(\stackrel{\circ}{W}_{f}^{(l)}\left(T_{1}\right) ; \stackrel{\circ}{W}_{u}^{(l)}\left(T_{1}\right)\right)}
\end{aligned}
$$

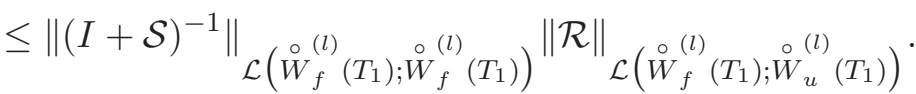

If we take $T_{1}$ so small, say, $\tau_{0}$, that $\|\mathcal{S}\|_{\mathcal{L}\left(\stackrel{\circ}{W}_{f}^{(l)}\left(T_{10}\right) ; W_{f}^{(l)}\left(\tau_{0}\right)\right)} \leq \frac{1}{2}$, then $\|(I+$ $\mathcal{S})^{-1} \|_{\mathcal{L}\left(\stackrel{\circ}{W}_{f}^{(l)}\left(\tau_{0}\right) ; \stackrel{\circ}{W}_{f}^{(l)}\left(\tau_{0}\right)\right)} \leq 2$, and

$$
\left\|\mathcal{A}^{-1}\right\|_{\mathcal{L}\left(\stackrel{\circ}{W}_{f}^{(l)}\left(\tau_{0}\right) ; \stackrel{\circ}{W_{u}^{(l)}}\left(\tau_{0}\right)\right)} \leq 2\|\mathcal{R}\|_{\mathcal{L}\left(\stackrel{\circ}{W}_{f}^{(l)}\left(\tau_{0}\right) ; \stackrel{\circ}{W}_{u}^{(l)}\left(\tau_{0}\right)\right)} .
$$

Note that the right-hand side of above inequality is estimated by Lemma 1. This completes the proof of Theorem 4 .

\subsection{Proof of Theorem 3}

Now, we are in a position to prove Theorem 3. We first state that $\boldsymbol{u}^{(j)}$ constructed in Section 5.2 satisfies $\boldsymbol{u}^{(j)} \in W_{2}^{l-1-2 j}(I)$ and

$$
\left\|\boldsymbol{u}^{(j)}\right\|_{W_{2}^{l-1-2 j}(I)} \leq c_{510}\left(\left\|\boldsymbol{f}_{1}\right\|_{W_{2}^{l-2, \frac{l-2}{2}}\left(I_{T_{1}}\right)}+\left\|\widetilde{\boldsymbol{u}}_{0}\right\|_{W_{2}^{l-1}(I)}\right) .
$$

Actually, in case $j=1$, we have

$$
\begin{aligned}
\left\|\boldsymbol{u}^{(1)}\right\|_{W_{2}^{l-3}(I)} & \leq\left\|L \widetilde{\boldsymbol{u}}_{0}\right\|_{W_{2}^{l-3}(I)}+\left\|\left.\boldsymbol{f}_{1}\right|_{t=0}\right\|_{W_{2}^{l-3}(I)} \\
& \leq\left\|\widetilde{\boldsymbol{u}}_{0}\right\|_{W_{2}^{l-1}(I)}+\left\|\boldsymbol{f}_{1}\right\|_{W_{2}^{l-2, \frac{l-2}{2}}\left(I_{T_{1}}\right)} .
\end{aligned}
$$

In case $j \geq 1$, we have

$$
\begin{aligned}
\left\|\boldsymbol{u}^{(j)}\right\|_{W_{2}^{l-1-2 j}(I)} \leq\left\|\left.\boldsymbol{f}_{1}\right|_{t=0}\right\|_{W_{2}^{l-1-2 j}(I)} & +\sum_{k=0}^{j-1}{ }_{j-1} C_{k}\left\|L^{(j-1-k)} \boldsymbol{u}^{(k)}\right\|_{W_{2}^{l-1-2 j}(I)} \\
& \leq\left\|\boldsymbol{f}_{1}\right\|_{W_{2}^{l-2 j, \frac{l-2 j}{2}}\left(I_{T_{1}}\right)}+c_{511} \sum_{k=0}^{j-1}\left\|\boldsymbol{u}^{(k)}\right\|_{W_{2}^{l-1-2(j-1)}(I)} \\
& \leq\left\|\boldsymbol{f}_{1}\right\|_{W_{2}^{l-2 j, \frac{l-2 j}{2}}\left(I_{T_{1}}\right)}+c_{511} \sum_{k=0}^{j-1}\left\|\boldsymbol{u}^{(k)}\right\|_{W_{2}^{l-1-2 k}(I)} .
\end{aligned}
$$


Thereby, the estimate of $\left\|\boldsymbol{u}^{(j)}\right\|_{W_{2}^{l-1-2 j}(I)}$ reduces to the estimates of

$$
\left\{\left\|\boldsymbol{u}^{(k)}\right\|_{W_{2}^{l-1-2 k}(I)}\right\}_{k=0}^{j-1} .
$$

But $\boldsymbol{u}^{(0)}=\widetilde{\boldsymbol{u}}_{0}$ and together with (24), (25), we arrive at (23).

In virtue of the well-known method, it is possible to construct a function $\boldsymbol{v}$ that satisfies (10) and the following inequality (see, for instance, Theorem IV.4.3 in [9], p.298):

$$
\begin{aligned}
\|\boldsymbol{v}\|_{W_{2}^{l, 2}}^{l, \frac{l}{2}\left(\mathbf{R}_{T_{1}}\right)} & \leq c_{512} \sum_{j=0}^{[(l / 2]}\left\|\hat{\boldsymbol{u}}^{(j)}\right\|_{W_{2}^{l-2 j}(\mathbf{R})} \\
& \leq c_{513} \sum_{j=0}^{[(l / 2]}\left\|\boldsymbol{u}^{(j)}\right\|_{W_{2}^{l-2 j}(I)} \\
& \leq c_{514}\left(\left\|\boldsymbol{f}_{1}\right\|_{W_{2}^{l-2, \frac{l-2}{2}}\left(I_{T_{1}}\right)}+\left\|\widetilde{\boldsymbol{u}}_{0}\right\|_{W_{2}^{l-1}(I)}\right) .
\end{aligned}
$$

Here, $\hat{\boldsymbol{u}}^{(j)}$ are the extension of $\boldsymbol{u}^{(j)}$ onto $\boldsymbol{R}$ satisfying

$$
\left\|\hat{\boldsymbol{u}}^{(j)}\right\|_{W_{2}^{l-1-2 j}(\mathbf{R})} \leq c_{515}\left\|\boldsymbol{u}^{(j)}\right\|_{W_{2}^{l-1-2 j}(I)} .
$$

Now, for $\tau_{0}$ derived in Theorem 4 , we have the estimate of $\widetilde{\boldsymbol{u}}$, which is the solution to (8), as follows:

$$
\begin{aligned}
\|\widetilde{\boldsymbol{u}}\|_{W_{u}^{(l)}\left(\tau_{0}\right)} \leq & \|\breve{\boldsymbol{u}}\|_{W_{u}^{(l)}\left(\tau_{0}\right)}+\|\boldsymbol{v}\|_{W_{2}^{l, \frac{l}{2}}\left(I_{\tau_{0}}\right)} \\
\leq \| & \left\|\widetilde{\boldsymbol{u}}_{0}\right\|_{W_{2}^{l-1}(I)}+\left\|\boldsymbol{f}_{1}^{\prime}\right\|_{W_{2}^{l-2, \frac{l-2}{2}}}{ }_{\left(I_{\tau_{0}}\right)} \\
& +\left\|\boldsymbol{f}_{1}\right\|_{W_{2}^{l-2, \frac{l-2}{2}}\left(I_{\tau_{0}}\right)}+\left\|F_{2}^{\prime}\right\|_{W_{2}^{\frac{l}{2}-\frac{3}{4}}\left(0, \tau_{0}\right)} \\
\leq & \left\|\widetilde{\boldsymbol{u}}_{0}\right\|_{W_{2}^{l-1}(I)}+\left\|F_{1}\right\|_{W_{2}^{l-2, \frac{l-2}{2}}\left(I_{\tau_{0}}\right)}+\left\|F_{2}\right\|_{W_{2}^{\frac{l}{2}-\frac{3}{4}}\left(0, \tau_{0}\right)} .
\end{aligned}
$$

Since $\boldsymbol{u} \in W_{u}^{(l)}\left(\tau_{0}\right)$, we have

$$
\left.\phi^{(j)}(x) \equiv \frac{\partial^{j} \boldsymbol{u}}{\partial t^{j}}\right|_{t=\frac{\tau_{0}}{2}} \in W_{2}^{l-2 j}(I) \quad(j=1,2, \ldots,[l / 2]) .
$$

Then, we can construct a function $\boldsymbol{v}^{\prime} \in W_{2}^{l, \frac{l}{2}}\left(\mathbf{R}_{T_{1}}\right)$ in such a way that

$$
\left.\frac{\partial^{j} \boldsymbol{v}^{\prime}}{\partial t^{j}}\right|_{t=\frac{\tau_{0}}{2}}=\phi^{(j)}(x) \quad \text { on } I \quad(j=0,1,2, \ldots,[l / 2]),
$$


and

$$
\left\|\boldsymbol{v}^{\prime}\right\|_{W_{2}^{l, \frac{l}{2}}\left(I_{T}\right)} \leq c_{516} \sum_{j=0}^{[l / 2]}\left\|\phi^{(j)}\right\|_{W_{2}^{(l-2 j)}(I)}
$$

hold. This is achieved by the same way as we constructed $\boldsymbol{v}$ earlier. Then, the function $\widetilde{\boldsymbol{u}}^{\prime \prime} \equiv \widetilde{\boldsymbol{u}}-\boldsymbol{v}^{\prime}$ satisfies

$$
\left\{\begin{array}{l}
\frac{\partial \widetilde{\boldsymbol{u}}^{\prime \prime}}{\partial t}-L\left(x, \frac{\partial}{\partial x}\right) \widetilde{\boldsymbol{u}}^{\prime \prime}=\boldsymbol{f}_{1}-L\left(x, \frac{\partial}{\partial x}\right) \widetilde{\boldsymbol{v}}^{\prime} \equiv \boldsymbol{f}_{1}^{\prime \prime} \text { in } I, \\
B\left(x, \frac{\partial}{\partial x}\right) \widetilde{\boldsymbol{u}}^{\prime \prime}=F_{2}-B\left(x, \frac{\partial}{\partial x}\right) \widetilde{\boldsymbol{v}}^{\prime} \equiv F_{2}^{\prime \prime} \text { on } \partial I, \\
\left.\widetilde{\boldsymbol{u}}^{\prime \prime}\right|_{t=\frac{\tau_{0}}{2}}=\mathbf{0} \text { on } I .
\end{array}\right.
$$

Again, in virtue of Theorem 4, we can assert that (27) has a solution $\widetilde{\boldsymbol{u}}^{\prime \prime}$ on $\left(\frac{\tau_{0}}{2}, \frac{3 \tau_{0}}{2}\right)$, which satisfies

$$
\left\|\widetilde{\boldsymbol{u}}^{\prime \prime}\right\|_{W_{u}^{(l)}\left(\frac{\tau_{0}}{2}, \frac{3 \tau_{0}}{2}\right)} \leq\left\|\boldsymbol{f}_{1}^{\prime \prime}\right\|_{W_{2}^{l-2, \frac{l-2}{2}}\left(I \times\left(\frac{\tau_{0}}{2}, \frac{3 \tau_{0}}{2}\right)\right)}+\left\|F_{2}^{\prime \prime}\right\|_{W_{2}^{\frac{l}{2}-\frac{3}{4}}\left(\frac{\tau_{0}}{2}, \frac{3 \tau_{0}}{2}\right)} .
$$

Thus, problem (8) has a solution $\widetilde{\boldsymbol{u}}$ on $\left(0, \frac{3 \tau_{0}}{2}\right)$, which satisfies

$$
\begin{gathered}
\|\widetilde{\boldsymbol{u}}\|_{W_{u}^{(l)}\left(\frac{3 \tau_{0}}{2}\right)} \leq\left\|\boldsymbol{f}_{1}^{\prime \prime}\right\|_{W_{2}^{l-2, \frac{l-2}{2}}\left(I_{\frac{3 \tau_{0}}{2}}\right)}+\left\|F_{2}^{\prime \prime}\right\|_{W_{2}^{\frac{l}{2}-\frac{3}{4}}\left(0, \frac{3 \tau_{0}}{2}\right)} \\
+\left\|\boldsymbol{u}_{0}\right\|_{W_{2}^{l-1}(I)} .
\end{gathered}
$$

By iterating this procedure repeatedly, we can extend the upper time of existence up to the desired $T>0$. This completes the proof of Theorem 3 .

\subsection{Proof of Theorem 2}

Now we are in a position to prove Theorem 2. First, we prepare some lemmas.

\subsection{Nonlinear Problem}

Next, we consider the nonlinear problem. Before proceeding to the detailed arguments, we prepare some lemmas. The following lemma is well known (see, for instance, [17].)

Lemma 2. Let $r \in(1 / 2,1)$. Then, the following issues hold: 
(i) For $f, g \in W_{2}^{1+r}(I)$,

$$
\|f g\|_{W_{2}^{1+r}(I)} \leq c_{517}\|f\|_{W_{2}^{1+r}(I)}\|g\|_{W_{2}^{1+r}(I)} .
$$

(ii) For $f \in W_{2}^{1+r}(I)$ and $g \in W_{2}^{r}(I)$ in general,

$$
\|f g\|_{W_{2}^{r}(I)} \leq c_{518}\|f\|_{W_{2}^{1+r}(I)}\|g\|_{W_{2}^{r}(I)} .
$$

Next, we state the following lemma to evaluate nonlinear terms.

Lemma 3. Assume that $l>5 / 2$, and $\widetilde{C}_{a 0}, \widetilde{C}_{r 0} \in W_{2}^{l}(I)$. Let $\widetilde{\boldsymbol{u}}=$ $\left(\widetilde{C}_{b}, \widetilde{C}_{a}, \widetilde{C}_{r}\right)^{\mathrm{T}}$ be a solution to $(5)$. Then, for arbitrary $T>0$, the following estimates hold with a certain $\varepsilon>0$.

$$
\begin{aligned}
& \left\|\frac{\partial}{\partial x}\left[\widetilde{C}_{b}^{(n)} \frac{\partial \widetilde{C}_{a}^{(n)}}{\partial x}\right]\right\|_{W_{2}^{l-2, \frac{l-2}{2}}\left(I_{T}\right)}^{2} \leq\left(\varepsilon+C_{\varepsilon} T\right)\left\|\widetilde{C}_{b}^{(n)}\right\|_{W_{2}^{l, \frac{l}{2}}\left(I_{T}\right)}^{2}+c_{512}, \\
& \left\|\frac{\partial}{\partial x}\left[\widetilde{C}_{b}^{(n)} \frac{\partial \widetilde{C}_{r}^{(n)}}{\partial x}\right]\right\|_{W_{2}^{l-2, \frac{l-2}{2}}\left(I_{T}\right)}^{2} \leq\left(\varepsilon+C_{\varepsilon} T\right)\left\|\widetilde{C}_{b}^{(n)}\right\|_{W_{2}^{l, \frac{l}{2}}\left(I_{T}\right)}^{2}+c_{513} .
\end{aligned}
$$

Here, $C_{\varepsilon}>0$ is a constant that depends on $\varepsilon$ decreasingly.

Proof. By $(5)_{2}$, we have

$$
\begin{gathered}
\frac{\partial \widetilde{C}_{a}^{(n)}}{\partial x}=\mathrm{e}^{-k_{a} t} \frac{\partial \widetilde{C}_{a 0}}{\partial x}+a_{1}(x) \int_{0}^{t} \mathrm{e}^{-k_{a}(t-s)} \frac{\partial \widetilde{C}_{b}^{(n)}}{\partial x}(x, s) \mathrm{d} s \\
+a_{1}^{\prime}(x) \int_{0}^{t} \mathrm{e}^{-k_{a}(t-s)} \frac{\partial \widetilde{C}_{b}^{(n)}}{\partial x}(x, s) \mathrm{d} s
\end{gathered}
$$

Therefore, we have

$$
\begin{aligned}
\frac{\partial}{\partial x}\left[\widetilde{C}_{b}^{(n)} \frac{\partial \widetilde{C}_{a}^{(n)}}{\partial x}\right] & =\frac{\partial \widetilde{C}_{b}^{(n)}}{\partial x}\left\{\mathrm{e}^{-k_{a} t} \frac{\partial \widetilde{C}_{a 0}}{\partial x}\right. \\
& \left.+a_{1}(x) \int_{0}^{t} \mathrm{e}^{-k_{a}(t-s)} \frac{\partial \widetilde{C}_{b}^{(n)}}{\partial x}(x, s) \mathrm{d} s\right\} \\
& +a_{1}^{\prime} \int_{0}^{t} \mathrm{e}^{-k_{a}(t-s)} \frac{\partial \widetilde{C}_{b}^{(n)}}{\partial x}(x, s) \mathrm{d} s
\end{aligned}
$$




$$
\begin{gathered}
+\widetilde{C}_{b}\left\{\mathrm{e}^{-k_{a} t} \frac{\partial^{2} \widetilde{C}_{a 0}}{\partial x^{2}}+a_{1}^{\prime \prime}(x) \int_{0}^{t} \mathrm{e}^{-k_{a}(t-s)} \frac{\partial \widetilde{C}_{b}^{(n)}}{\partial x}(x, s) \mathrm{d} s\right. \\
+2 a_{1}^{\prime}(x) \int_{0}^{t} \mathrm{e}^{-k_{a}(t-s)} \frac{\partial^{2} \widetilde{C}_{b}^{(n)}}{\partial x^{2}}(x, s) \mathrm{d} s \\
\left.+a_{1}(x) \int_{0}^{t} \mathrm{e}^{-k_{a}(t-s)} \frac{\partial^{2} \widetilde{C}_{b}^{(n)}}{\partial x^{2}}(x, s) \mathrm{d} s\right\} .
\end{gathered}
$$

We shall show the estimates of some terms in (28). By the assumption and Lemma 2, it is easy to see

$$
\left\|\frac{\partial^{2} \widetilde{C}_{a 0}}{\partial x^{2}} \widetilde{C}_{b}^{(n)}(t)\right\|_{W_{2}^{l-2}(I)}^{2} \leq c_{519}\left\|\frac{\partial^{2} \widetilde{C}_{a 0}}{\partial x^{2}}\right\|_{W_{2}^{l-2}(I)}^{2}\left\|\widetilde{C}_{b}^{(n)}(t)\right\|_{W_{2}^{l-1}(I)}^{2} .
$$

Therefore, by using the interpolation inequality of the Sobolev-Slobodetskil spaces, we have

$$
\begin{array}{rl}
\int_{0}^{T_{1}}\left\|\frac{\partial^{2} \widetilde{C}_{a 0}}{\partial x^{2}} \widetilde{C}_{b}^{(n)}(t)\right\|_{W_{2}^{l-2}(I)}^{2} & \mathrm{~d} t \\
\leq & \leq c_{520}\left\|\frac{\partial^{2} \widetilde{C}_{a 0}}{\partial x^{2}}\right\|_{W_{2}^{l-2}(I)}^{2}\left\|\widetilde{C}_{b}^{(n)}\right\|_{L_{2}\left(0, T_{1} ; W_{2}^{l-1}(I)\right)}^{2} \\
\leq\left(\varepsilon+C_{\varepsilon} T_{1}\right)^{2}\left\|\widetilde{C}_{b}^{(n)}\right\|_{W_{2}^{l, \frac{l}{2}}\left(I_{T_{1}}\right)}^{2}
\end{array}
$$

Next, we show the estimate of

$$
\left\|\widetilde{C}_{b}^{(n)} a_{1}^{\prime}(x) \int_{0}^{t} \mathrm{e}^{-k_{a}(t-s)} \frac{\partial \widetilde{C}_{b}^{(n)}}{\partial x}(x, s) \mathrm{d} s\right\|_{W_{2}^{l-2, \frac{l-2}{2}}\left(I_{T}\right)} .
$$

Thanks to Lemma 2 again, we have

$$
\begin{aligned}
& \left\|\widetilde{C}_{b}^{(n)}(\cdot, t) \int_{0}^{t} \mathrm{e}^{-k_{a}(t-s)} \frac{\partial \widetilde{C}_{b}^{(n)}}{\partial x}(\cdot, s) \mathrm{d} s\right\|_{W_{2}^{l-2}(I)} \\
& \quad \leq\left\|\widetilde{C}_{b}^{(n)}(t)\right\|_{W_{2}^{l-1-\delta}(I)}\left\|\int_{0}^{t} \mathrm{e}^{-k_{a}(t-s)} \frac{\partial \widetilde{C}_{b}^{(n)}}{\partial x}(\cdot, s) \mathrm{d} s\right\|_{W_{2}^{l-2}(I)},
\end{aligned}
$$


with $\delta>0$ sufficiently small. Then,

$$
\begin{array}{r}
\int_{0}^{T_{1}}\left\|\widetilde{C}_{b}^{(n)}(t)\right\|_{W_{2}^{l-1-\delta}(I)}^{2}\left\|\int_{0}^{t} \mathrm{e}^{-k_{a}(t-s)} \frac{\partial \widetilde{C}_{b}^{(n)}}{\partial x}(\cdot, s) \mathrm{d} s\right\|_{W_{2}^{l-2}(I)}^{2} \mathrm{~d} t \\
\leq \sup _{t \in\left(0, T_{1}\right)}\left\|\widetilde{C}_{b}^{(n)}(t)\right\|_{W_{2}^{l-1-\delta}(I)}^{2} \\
\quad \times \int_{0}^{T_{1}}\left\|\int_{0}^{t} \mathrm{e}^{-k_{a}(t-s)} \frac{\partial \widetilde{C}_{b}^{(n)}}{\partial x}(\cdot, s) \mathrm{d} s\right\|_{W_{2}^{l-2}(I)}^{2} \mathrm{~d} t .
\end{array}
$$

By the Cauchy-Schwarz inequality, we have

$$
\left\|\int_{0}^{t} \mathrm{e}^{-k_{a}(t-s)} \frac{\partial \widetilde{C}_{b}^{(n)}}{\partial x}(\cdot, s) \mathrm{d} s\right\|_{W_{2}^{l-2}(I)}^{2} \leq \frac{1}{2 k_{a}}\left\|\frac{\partial \widetilde{C}_{b}^{(n)}}{\partial x}\right\|_{W_{2}^{l-2,0}\left(I_{t}\right)}^{2} .
$$

Thus, with the aid of the Sobolev embedding theorem and the interpolation inequality, the right-hand side of (27) is estimated by

$$
\begin{aligned}
\sup _{t \in\left(0, T_{1}\right)}\left\|\widetilde{C}_{b}^{(n)}(t)\right\|_{W_{2}^{l-1-\delta}(I)}^{2} & \times \frac{1}{2 k_{a}} \int_{0}^{T_{1}}\left\|\frac{\partial \widetilde{C}_{b}^{(n)}}{\partial x}(\cdot, s)\right\|_{W_{2}^{l-2}(I)}^{2} \mathrm{~d} s \\
\leq & T_{1}\left(\varepsilon+C_{\varepsilon} T_{1}\right)^{2}\left\|\widetilde{C}_{b}^{(n)}\right\|_{W_{2}^{l, \frac{l}{2}}\left(I_{T_{1}}\right)}^{4}
\end{aligned}
$$

Other terms in the right-hand side of (28) are estimated similarly. These yield the desired first inequality of Lemma 3 . The second one is estimated in a similar manner.

Now, we prove Theorem 2. We define a successive sequence $\left\{\widetilde{\boldsymbol{u}}^{(n)}\right\}_{n=0}^{+\infty}$ with $\widetilde{\boldsymbol{u}}^{(n)}=\left(\widetilde{C}_{b}^{(n)}, \widetilde{C}_{a}^{(n)}, \widetilde{C}_{r}^{(n)}\right)^{\mathrm{T}}$. For $n=0$, we define $\widetilde{\boldsymbol{u}}^{(n)}=\widetilde{\boldsymbol{u}}_{0}$. For $n \geq 1$, we 
define it inductively:

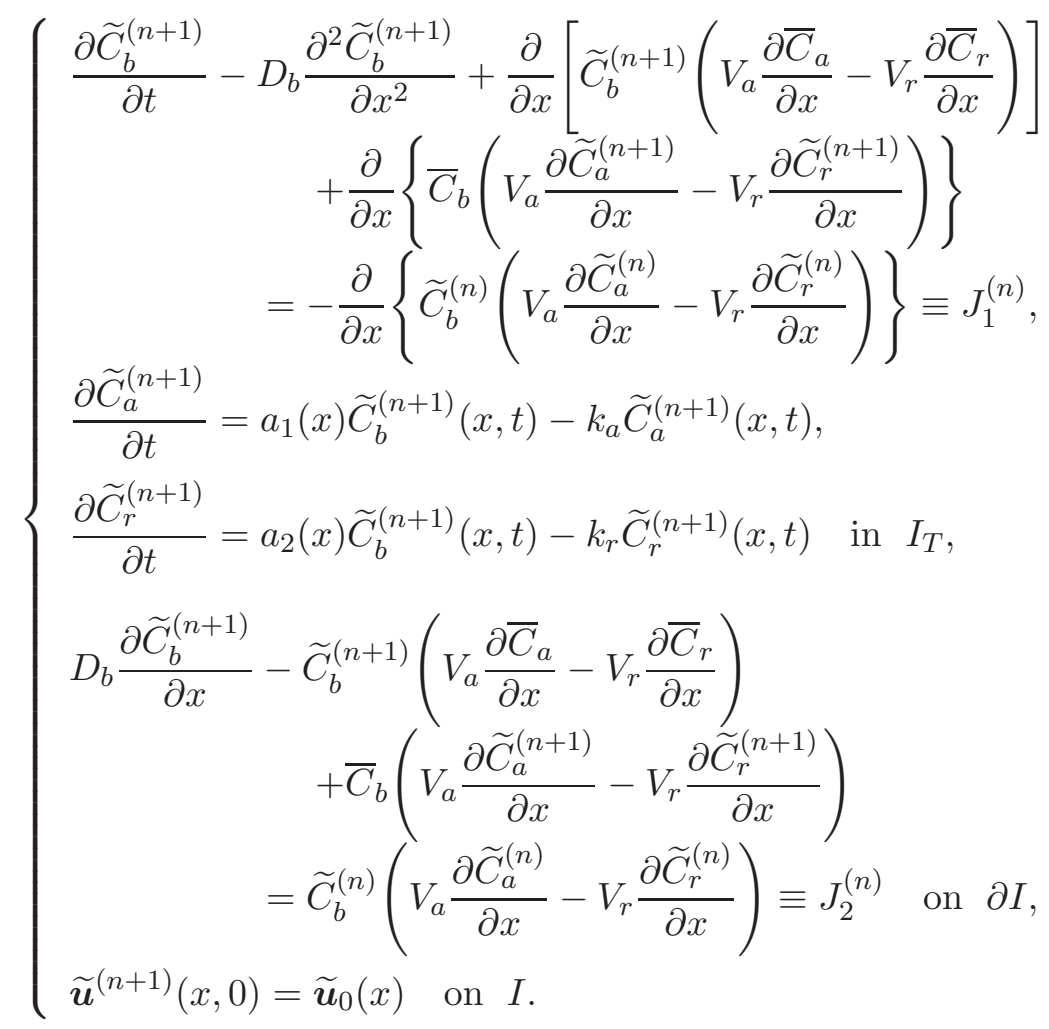

We also introduce notions $E_{0} \equiv\left\|\widetilde{\boldsymbol{u}}_{0}\right\|_{W_{2}^{l-1}(I)}$, and $E_{(n)}(t) \equiv\left\|\widetilde{\boldsymbol{u}}^{(n)}\right\|_{W_{u}^{(l)}(t)}(n \geq 1)$. Then, by virtue of Theorem 4 , we have the estimate

$$
E_{(n+1)}(t) \leq c_{521}\left(\left\|J_{1}^{(n)}\right\|_{W_{2}^{l-2, \frac{l-2}{2}}\left(I_{T_{1}}\right)}+\left\|J_{2}^{(n)}\right\|_{W_{2}^{\frac{l}{2}-\frac{3}{4}}\left(0, T_{1}\right)}+\left\|\widetilde{\boldsymbol{u}}_{0}\right\|_{W_{2}^{l-1}(I)}\right) .
$$

By applying Lemma 3 to the estimates of nonlinear terms, we have

$$
E_{(n+1)}(t) \leq c_{522}\left(\varepsilon+C_{\varepsilon} T_{2}\right) \phi_{51}\left(E_{(n)}(t)\right)+c_{523} .
$$

Here, $\phi_{51}(\cdot)$ is a homogeneous polynomial of at most two degree of its argument. Take a constant $M_{51}$ such that $M_{51}>E_{0}$ holds. Then, assume that

$$
E_{(n)}(t) \leq M_{51} \text {. }
$$

holds for a certain $n$. Take $\varepsilon$ first so that

$$
\varepsilon c_{522} \phi_{51}\left(M_{51}\right)+c_{523}<M_{51}
$$


holds, and then $T_{51}$ small enough so that

$$
c_{522} C_{\varepsilon} T_{51} \phi_{51}\left(M_{51}\right)<M_{51}-\varepsilon c_{522} \phi_{51}\left(M_{51}\right)-c_{523}
$$

holds. Then, we obtain

$$
E_{(n+1)}\left(T_{51}\right) \leq M_{51}
$$

from the assumption $E_{(n)}\left(T_{51}\right) \leq M_{51}$. Thus, by induction, the sequence $\left\{\widetilde{\boldsymbol{u}}^{(n)}\right\}_{n}$ is well-defined in $W_{u}^{(l)}\left(T_{51}\right)$.

Next, we have to verify it certainly converges in $W_{u}^{(l)}\left(T_{52}\right)$ with a certain $T_{52} \in\left(0, T_{51}\right]$. In order to show that, we define $\breve{\boldsymbol{u}}^{(n)}=\left(\breve{C}_{b}^{(n)}, \breve{C}_{a}^{(n)}, \breve{C}_{r}^{(n)}\right)^{\mathrm{T}} \equiv$ $\widetilde{\boldsymbol{u}}^{(n)}-\widetilde{\boldsymbol{u}}^{(n-1)}$ for $n \geq 1$, and subtract (30) with $n$ replaced with $n-1$ from itself. Then, we consider the following problem:

$$
\left\{\begin{array}{l}
\frac{\partial \breve{C}_{b}^{(n+1)}}{\partial t}-D_{b} \frac{\partial^{2} \breve{C}_{b}^{(n+1)}}{\partial x^{2}}+\frac{\partial}{\partial x}\left[\breve{C}_{b}^{(n+1)}\left(V_{a} \frac{\partial \bar{C}_{a}}{\partial x}-V_{r} \frac{\partial \bar{C}_{r}}{\partial x}\right)\right] \\
+\frac{\partial}{\partial x}\left\{\bar{C}_{b}\left(V_{a} \frac{\partial \breve{C}_{a}^{(n+1)}}{\partial x}-V_{r} \frac{\partial \breve{C}_{r}^{(n+1)}}{\partial x}\right)\right\}=J_{1}^{(n)}-J_{1}^{(n-1)} \\
\frac{\partial \breve{C}_{a}^{(n+1)}}{\partial t}=a_{1}(x) \breve{C}_{b}^{(n+1)}(x, t)-k_{a} \breve{C}_{a}^{(n+1)}(x, t), \\
\frac{\partial \breve{C}_{r}^{(n+1)}}{\partial t}=a_{2}(x) \breve{C}_{b}^{(n+1)}(x, t)-k_{r} \breve{C}_{r}^{(n+1)}(x, t) \quad \text { in } I, \\
D_{b} \frac{\partial \breve{C}_{b}^{(n+1)}}{\partial x}-\breve{C}_{b}^{(n+1)}\left(V_{a} \frac{\partial \bar{C}_{a}}{\partial x}-V_{r} \frac{\partial \bar{C}_{r}}{\partial x}\right) \\
-\bar{C}_{b}\left(V_{a} \frac{\partial \breve{C}_{a}^{(n)}}{\partial x}-V_{r} \frac{\partial \breve{C}_{r}^{(n)}}{\partial x}\right)=J_{2}^{(n)}-J_{2}^{(n-1)} \text { on } \partial I, \\
\breve{\boldsymbol{u}}^{(n)}(x, 0)=\mathbf{0} \text { on } I .
\end{array}\right.
$$

Introduce a notion $\breve{E}_{(n)}(t) \equiv\left\|\breve{\boldsymbol{u}}^{(n)}\right\|_{W_{u}^{(l)}(t)}$. Then, by virtue of Theorem 3, we have the estimate

$$
\breve{E}_{(n+1)}(t) \leq c_{524}\left(\varepsilon+C_{\varepsilon} t\right) \phi_{52}\left(E_{(n-1)}(t), E_{(n)}(t)\right) \breve{E}_{(n)}(t) .
$$

Take $\varepsilon$ sufficiently small first so that

$$
c_{524} \varepsilon \phi_{52}\left(M_{51}, M_{51}\right)<1
$$

holds, and then $T_{52}$ so that

$$
c_{524} C_{\varepsilon} T_{52} \phi_{52}\left(M_{51}, M_{51}\right)<1-c_{524} \varepsilon \phi_{52}\left(M_{51}, M_{51}\right)
$$


holds with this $\varepsilon$. For these $\varepsilon$ and $T_{52}$, we have

$$
\breve{E}_{(n+1)}\left(T_{52}\right) \leq d \breve{E}_{(n)}\left(T_{52}\right), \quad d=c_{524}\left(\varepsilon+C_{\varepsilon} T_{52}\right) \phi_{52}\left(M_{52}, M_{52}\right) \in(0,1) .
$$

Therefore, we can conclude that $\left\{\widetilde{\boldsymbol{u}}^{(n)}\right\}_{n}$ forms a Cauchy sequence in $W_{u}^{(l)}\left(T_{52}\right)$. Thus, the limitation

$$
\widetilde{\boldsymbol{u}} \equiv \lim _{n \rightarrow+\infty} \widetilde{\boldsymbol{u}}_{n}
$$

exists in the same function space, which is our desired solution. This $T_{52}$ is the very $T_{*}$ we have seeked for. Uniqueness is easily seen by the similar argument, and we omit it here. These complete the proof of Theorem 2.

\section{Conclusion}

In this paper, we provided the local-in-time solvability of the one-dimensional non-stationary problem of the target detection model in the molecular communication network, in the Sobolev-Slobodetskiu space. We shall extend our study to the two-dimensional case, and also consider the stability arguments in the near future.

\section{References}

[1] A. Einolghozati, M. Sardari, A. Beirami and F. Fekri, Capacity of discrete molecular diffusion channels, In: Proc. IEEE International Symposium on Information Theory (2011).

[2] A. Einolghozati, M. Sardari and F. Fekri, Capacity of diffusion-based molecular communication with ligand receptors, In: Proc. IEEE Information Theory Workshop (2011).

[3] T. Hillen and A. P. Potapov, The one-dimensional chemotaxis model : global existence and asymptotic profile, Math. Methods Appl. Sci., 27 (2004), 1783-1801.

[4] D. Horstmann, From 1970 until present: the Keller-Segel model in chemotaxis and its consequences, I., Jahresber. Dtsch. Math.-Verein., 105 (2003), 103-165.

[5] S. Iwasaki, Convergence of solutions to simplified self-organizing targetdetection model, Sci. Math. Jpnonicae. e-2016 (2016). 
[6] S. Iwasaki and T. Bak, A mathematical model of non-diffusion-based mobile molecular communication networks, IEEE Comm. Lettr., 65 (2017), 203-230.

[7] K. Kang and T. Kolokolnikov, The stability and dynamics of a spike in the 1D Keller-Segel model, IMA J. Appl. Math., 72 (2007), 140-162.

[8] E. F. Keller and L. A. Segel, Model for chemotaxis, J. Theor. Biol., 30 (1971), 225-234.

[9] O. A. Ladyženskaja, V. A. Solonnikov and N. N. Ural'ceva, Linear and Quasi-linear Equations of Parabolic Type, Translations of Mathematical Monographs 23, American Mathematical Society (1968).

[10] T. Nakano, et al., Molecular Communication, Cambridge University Press, Cambridge (2013).

[11] T. Nakano, et al., Performance evaluation of leader-follower-based mobile molecular communication networks for target detection applications, IEEE Trans. Comm., 65 (2017), 663-676.

[12] Y. Okaie, et al. Modeling and performance evaluation of mobile bionanocensor networks for target tracking, In: Proc. IEEE ICC, (2014), 3969-3974.

[13] Y. Okaie, et al. Cooperative target tracking by a mobile bionanosensor network, IEEE Trans. Nanobioscience, 13 (2014), 267-277.

[14] K. Osaki and A. Yagi, A Finite dimensional attractor for one-dimensional Keller-Segel equations, Funkcialaj Ekvacioj, 44 (2001), 441-469.

[15] R. Schaaf, Stationary solutions of chemotaxis systems, Trans. Amer. Math. Soc., 292 (1985), 531-556.

[16] A. Marciniak-Czochra, G. Karch and K. Suzuki, Instability of turing patterns in reaction-diffusion-ODE systems, J. Math.Biol., 74 (2017), 583618.

[17] N. Tanaka and A. Tani, Surface waves for a compressible viscous fluid, J. Math. Fluid Mech., 5 (2003), 303-363.

[18] J. Wloka, Partielle Differentialgleichungen, B. G. Teubner (1982). 
